\title{
Adjuvant chemotherapy for early stage non-small cell lung
}

\section{cancer}

\section{Manali I. Patel and Heather A. Wakelee*}

Oncology, Department of Medicine, Stanford Cancer Institute, Stanford University, Stanford, CA, USA

Edited by:

Masahiro Tsuboi, Kanagawa Cancer

Center, Japan

\section{Reviewed by:}

Solange Peters, Centre Hospitalier Universitaire Vaudois, Switzerland Sacha I. Rothschild, University

Hospital Basel, Switzerland

*Correspondence:

Heather A. Wakelee, Oncology, Stanford Cancer Institute, Stanford University, 875 Blake Wilbur, Stanford, CA 94305-5826, USA.

e-mail: hwakelee@stanford.edu
For many years adjuvant chemotherapy has been a standard treatment after complete resection in malignancies such as breast and colon but only recently has its use become standard in early stage non-small cell lung cancer (NSCLC). Although surgery is regarded as the best possible treatment for early stage NSCLC, only $20-25 \%$ of patients have resectable disease at presentation. Despite optimal surgical treatment, 5 -year survival rates for NSCLC remain $50-60 \%$ for stage IB, $40-50 \%$ for stage II, and $20-30 \%$ for stage III (Kohler et al., 2011; Siegel et al., 2011). Adjuvant chemotherapy provides additional survival benefit in resected NSCLC but questions remain as to how to select patients for therapy and which regimen is best. Other than work with tegafur/uracil in Japan, the positive adjuvant trials have all utilized a cisplatin backbone, but the drug(s) to pair with cisplatin are a matter of debate and will be discussed further in this manuscript.

Keywords: lung cancer, non-small cell, adjuvant, chemotherapy, early stage

\section{ADJUVANT CHEMOTHERAPY EMERGES FOR NSCLC}

The adoption of adjuvant chemotherapy for patients with early stage non-small cell lung cancer (NSCLC) is validated by three meta-analyses - the 1995 NSCLC Collaborative Group, the Medical Research Council (MRC), and the lung adjuvant cisplatin evaluation (LACE). The first large meta-analysis, the 1995 NSCLC Collaborative Group demonstrated a trend toward overall survival benefit in eight cisplatin-based adjuvant trials (Non-Small Cell Lung Cancer Collaborative Group, 1995). These results led to subsequent larger adjuvant trials utilizing cisplatin-based chemotherapy which were pooled in two recent large meta-analyses, the MRC and the LACE, both of which demonstrated a 5-year overall survival benefit (HR 0.87; 95\% CI 0.81-0.93; $p<0.000001$; HR 0.89; 95\% CI 0.82-0.96; $p=0.004$, respectively; Stewart et al., 2007; Pignon et al., 2008).

Five of the largest adjuvant trials to date were included in LACE; two exclusively examined cisplatin-vinorelbine combinations [National Cancer Institute of Canada (NCIC) JBR.10 and Adjuvant Navelbine International Trialist Association (ANITA)] while the other three allowed investigator-choice of cisplatin-based regimens [big lung trial (BLT), International Trialist Association Trial (IALT), and Adjuvant Lung Project Italy (ALPI)]. The adjuvant trial showing the most striking benefit, NCIC JBR.10, included 482 patients with completely resected IB or II NSCLC randomly assigned to observation or four cycles of weekly cisplatin $50 \mathrm{mg} / \mathrm{m}^{2}$ days 1,8 plus vinorelbine $25 \mathrm{mg} / \mathrm{m}^{2}$ days 1,8 , 15, 22 on a 28-day regimen (Winton et al., 2005). The ANITA trial evaluated adjuvant treatment for 840 patients with resected stage IB-IIIA NSCLC using cisplatin $100 \mathrm{mg} / \mathrm{m}^{2}$ day 1 (4 doses) plus vinorelbine at $30 \mathrm{mg} / \mathrm{m}^{2}$ days $1,8,15$, and 22 (16 doses) for four cycles versus observation (Douillard et al., 2006). Both trials demonstrated overall survival benefit ( $\mathrm{HR} 0.69, p=0.004 ; \mathrm{HR}$ $0.80, p=0.017$, respectively) and the survival advantage did not diminish over time $-8.4 \%$ at 7 years follow-up.
The BLT, IALT, and ALPI trials evaluated an investigator-chosen cisplatin-based regimen. The negative BLT utilized 3-week regimens with cisplatin/vindesine, mitomycin/ifosfamide/cisplatin, mitomycin/vinblastine/cisplatin, or vinorelbine/cisplatin (Waller et al., 2004). IALT, which showed overall survival benefit at 5 years (HR 0.86, p 0.03), but not at 7 years (HR 0.91, 95\% CI 0.81-1.02), enrolled 1867 patients and randomly assigned them to observation or to three or four cycles of one of the four following regimens: cisplatin plus etoposide, or a vinca alkaloid (vindesine, vinorelbine, or vinblastine; IALT, 2004). Finally, the negative ALPI trial utilized a regimen of three cycles of adjuvant cisplatin, mitomycin, and vindesine, dosed every 3 weeks (Scagliotti et al., 2003). Although only three trials (ANITA, IALT, JBR.10) independently demonstrated significant survival benefit ranging from 4 to $15 \%$ (HR 0.69-0.89), when pooled in LACE, cisplatin-based adjuvant therapy improved 5-year survival ranging from 4 to $5.3 \%(\mathrm{HR}=0.89$; $95 \%$ CI $0.82-0.96 ; p=0.004$ ). Owing to these encouraging results, MRC and LACE reaffirmed the benefit of adjuvant chemotherapy for stage II and IIIA but left questions regarding therapy for those with stage I disease. Many hypotheses have emerged about the differential survival seen in the trials such as inclusion of various stages, and differences in utilization of radiation therapy, but one of the most striking variances in the trials of course is the additional chemotherapy paired with cisplatin. It is possible that the additional agent(s) play a critical role in the amount of benefit provided by adjuvant chemotherapy.

\section{THE CISPLATIN VERSUS CARBOPLATIN DEBATE}

Cisplatin combinations are currently recommended as the backbone for adjuvant therapy given that all three positive trials after 1995 used a cisplatin-based regimen (two with vinorelbine; IALT, 2004; Winton et al., 2005; Douillard et al., 2006). Treatment with cisplatin, though, is associated with a number of serious and unpleasant side effects which, along with data demonstrating a 
role of carboplatin in metastatic settings, created the landscape for competition between cisplatin and carboplatin. The only large meta-analysis directly evaluating the two agents, albeit in advanced stage disease, the cisplatin versus carboplatin (CisCa) trial, provides evidence of a trend toward superiority of cisplatin over carboplatin (Ardizonni et al., 2007). CisCa demonstrated improved response rates with cisplatin therapy $(30 \%)$ compared with carboplatin (24\%) in 2968 patients (OR of 1.37; 95\% CI 1.16-1.62; $p<0.001)$. Subgroup analysis of patients with non-squamous tumors and those treated with third-generation chemotherapy further revealed that carboplatin-based chemotherapy was associated with a statistically significant increase in mortality $(\mathrm{HR}=1.12$; 95\% CI 1.01-1.23 and HR = 1.11; 95\% CI 1.01-1.21, respectively). Though these results will need to be updated with the recently presented BTOG2 Phase III randomized trial which compared carboplatin/gemcitabine to two different cisplatin/gemcitabine regimens and demonstrated equivalency of the carboplatin and cisplatin $\left(80 \mathrm{mg} / \mathrm{m}^{2}\right)$ arms, the majority of trials have still shown cisplatin superiority in the metastatic setting (Ferry et al., 2011). Our only data in early stage disease with carboplatin comes from the negative CALGB9633 trial which utilized carboplatin/paclitaxel for resected stage IB NSCLC (Strauss et al., 2008). Though this study failed to show a survival advantage in the population with the carboplatin regimen, the superiority of cisplatin may not be the only explanation given the small sample size and the exclusive inclusion of only stage IB. Based on the evidence to date, current National Comprehensive Cancer Network (NCCN) guidelines, an organization comprised of an alliance of professionals from 21 of the world's leading cancer centers, recommend adjuvant chemotherapy using a cisplatin-based regimen (Ettinger et al., 2010). Therefore as there are no further studies planned to evaluate this research question of carboplatin versus cisplatin, unless medically contraindicated, cisplatin is the platinum of choice in the adjuvant setting.

\section{IS THERE AN OPTIMAL REGIMEN?}

There are little data regarding what the best cisplatin-based regimen may be and though data is limited, many options are recommended by organizations such as the NCCN. Currently, the NCCN guidelines recommend combination cisplatin-based therapy with previously studied third-generation agents vinorelbine, vincristine, etoposide, but also suggest combinations with the newer agents gemcitabine, docetaxel, or pemetrexed which have not yet been studied extensively in the adjuvant setting. Pooled data from the LACE meta-analysis demonstrate a marginal trend toward survival using cisplatin/vinorelbine compared with other cisplatin combinations $(p=0.04)$. Of the five trials in LACE, the regimen with the most positive outcomes was cisplatin/vinorelbine (ANITA and JBR.10). One reason for this outcome may have more to do with delivery of the entire regimen and not necessarily due the vinorelbine itself. Eighty-six percent of the patients who received cisplatin-vinorelbine regimens were able to receive a higher cisplatin dosing $\left(>300 \mathrm{mg} / \mathrm{m}^{2}\right)$ compared with other regimens. It is possible that this dose intensity of cisplatin accounts for the differential benefit in the vinorelbine containing regimens. Currently, there are many modern drugs which have yet to be studied in the adjuvant setting. Our only definitive data, though, exists in the metastatic setting.
In the metastatic setting, the choice of therapy is a platinumbased doublet with third-generation agents such as paclitaxel, docetaxel, gemcitabine, vinorelbine, irinotecan, or pemetrexed. (Schiller et al., 2002; Baggstrom et al., 2007). Although the overall differential benefit from modifying chemotherapy in the metastatic setting has been small, the head-to-head comparisons of regimens of other doublets over cisplatin-vinorelbine are found in several studies. For instance, in the metastatic setting, the TAX 326 study group found that cisplatin-vinorelbine had a less favorable overall response and survival rate compared to cisplatin-docetaxel (24.5 versus $31.56 \%, p=0.029$; Fossella et al., 2003). Direct comparison of cisplatin-docetaxel versus cisplatin-gemcitabine have shown equivalency (Schiller et al., 2002). Comparisons of cisplatin-pemetrexed to cisplatin-gemcitabine demonstrate a superiority of cisplatin-pemetrexed in terms of survival (12.6 versus 10.9 months) as well as tolerability in patients with nonsquamous histology (Scagliotti et al., 2008). Therefore, extrapolating from these studies one could postulate that cisplatinpemetrexed is superior to cisplatin-gemcitabine in non-squamous patients and both are equivalent to cisplatin-taxane regimens and that all three combinations are superior to cisplatin-vinorelbine in the metastatic setting. Of course this is merely a stated opinion at this time, and further studies will be needed to confirm this hypothesis, but many including the authors are now comfortable using other platinum doublets besides cisplatin/vinorelbine as adjuvant therapy.

This is illustrated by the use of other platinum doublets as adjuvant therapy when allowed on clinical trial in the United States. From preliminary data of the Eastern Cooperative Oncology Group 1505 trial (which is currently underway to investigate the value in the adjuvant setting of the anti-vascular endothelial growth factor receptor monoclonal antibody bevacizumab) participating oncologists (primarily in the United States) are choosing widely from the combinations of treatment available on the trial which include four different cisplatin-based regimens: cisplatin with gemcitabine, docetaxel, vinorelbine, or pemetrexed. Out of the first 636 patients (of a planned 1500) enrolled in the trial, $27 \%$ received cisplatin + vinorelbine, $33 \%$ received cisplatin + docetaxel, $25 \%$ received cisplatin + gemcitabine and $16 \%$ received cisplatin + pemetrexed (only for non-squamous histology and added as an option after enrollment had already begun; Wakelee et al., 2010).

Tolerability of regimens however must be interpreted carefully in the adjuvant setting as was noted from the Memorial Sloan Kettering Cancer Center Phase II tolerability study of cisplatin plus docetaxel in the adjuvant setting in NSCLC. In this trial the side effect profile led to poor completion of treatment with $55 \%$ of patients failing to complete three cycles secondary to fatigue, nausea, neutropenic fevers, hypotension, and renal side effects (Azzoli et al., 2007). Further studies are similarly investigating the refinement of chemotherapy and tolerability in the adjuvant setting. Preliminary results from the randomized phase II trial on refinement of early stage NSCLC adjuvant chemotherapy with cisplatin and pemetrexed $(\mathrm{CPx})$ versus cisplatin and vinorelbine $(\mathrm{CVb}$; TREAT) was presented by Kreuter et al. (2011). This trial demonstrated the feasibility of use of cisplatin-pemetrexed and the safety of treatment as well as the higher dose density of this regimen compared with cisplatin-vinorelbine. Although the efficacy of 
cisplatin-pemetrexed compared to cisplatin-vinorelbine is yet to be finalized one of the limitations of this trial is that $45 \%$ of the patients included in the study have squamous histology and $38 \%$ have stage IB which may significantly reduce the overall efficacy of cisplatin-pemetrexed. For now, whether data can be translated from the metastatic setting to the adjuvant setting is unknown but data suggests that it is reasonable to conclude that substituting other cisplatin-based doublets for cisplatin-vinorelbine may be considered.

\section{ADJUVANT CHEMOTHERAPY IN THE ELDERLY}

Adjuvant therapy in the elderly is also a research area of particular importance given that the median age of diagnosis of NSCLC is 70 years of age. In general, elderly patients receive less adjuvant therapy but studies to date demonstrate that elderly can enjoy a survival benefit with treatment without any differences in toxicities, hospitalizations, or treatment-related death by age group. In the JBR.10 trial, out of 155 patients aged 65 years and older, adjuvant chemotherapy significantly prolonged overall survival (HR $0.61 ; 95 \%$ CI $0.38-0.98 ; p=0.04$; JBR 10). In LACE which included JBR.10, pooled analysis of $414(9 \%)$ patients age 70 years and older also demonstrated a trend toward survival benefit with adjuvant chemotherapy (HR 0.90; 95\% CI 0.70-1.16; $p=0.29$; Fruh et al., 2008). These findings have improved rates of chemotherapy administration in the elderly at least in the United States and Canada as demonstrated in two abstracts presented at the American Society of Clinical Oncology (ASCO) Annual meeting in 2011. A population based study from Ontario Canada demonstrated that administration of adjuvant therapy in patients over 70 increased from 3 to $16 \%$ over the years of 2001-2006 with an overall survival improvement from 47 to $50 \%$ (Cuffe et al., 2011). Despite concerns of toxicity from chemotherapy, $70 \%$ of elderly were treated with cisplatin versus $28 \%$ treated with carboplatin. Similarly, in the United States, SEER data from 1992 to 2005 demonstrate that $19 \%$ of patients greater than age 65 received chemotherapy in the adjuvant setting (Gu et al., 2011), but the use of carboplatin was extensive. The evidence of benefit of adjuvant therapy for patients age 66-74 is clear and treatment should be offered for those patients with good performance status.

\section{IMPROVING PATIENT SELECTION AND FUTURE DIRECTIONS}

Although the benefit of adjuvant therapy is undeniable, the improvement is modest. The role of predictive markers is crucial as they may help us better select patients most likely to benefit from adjuvant therapy. In the IALT trial, patients with excision of repair cross-complementation group 1 (ERCC1)-negative tumors showed prolonged survival (HR 0.65; 95\% CI 0.50-0.86; $p=0.002)$ compared with observation, however this was not true among patients with ERCC1 positive tumors ( $\mathrm{HR}=1.14 ; 95 \% \mathrm{CI}$ $0.84-1.55$; $p=0.40$; Olaussen et al., 2006). A recent update presented at ESMO 2011 demonstrated a final HR of 0.91 during the 8 -years of median follow-up and an observed 20\% more deaths among ERCC1 positive tumors with an OS (HR of 1.20; 95\% CI 0.91-1.59) among patients with ERCC1 positive tumors. Multiple trials ongoing in the US and Europe are seeking to validate these results prospectively including SWOG 0720 which evaluated adjuvant therapy with cisplatin/gemcitabine for stage $1(>2 \mathrm{~cm})$ tumors in patients with low tumor expression of ERCC1 and RRM1.

The Tailored Post-surgical therapy in early stage NSCLC (TASTE) trial, Spanish Customized Adjuvant Trial (SCAT), and International Tailored chemotherapy adjuvant trial (ITACA) are all phase III trials using four chemotherapy possibilities based on expression levels of ERCC1 and markers such as epidermal growth factor receptor (EGFR) mutations, thymidylate synthase expression and others. Gene expression patterns are also under investigation by multiple groups. The MAGRIT (MAGE-A3 as Adjuvant, Non-Small Cell Lung Cancer Immunotherapy) trial will evaluate the MAGE-A3 Antigen Specific Cancer Immunotherapeutic in the adjuvant setting and will hopefully shed light on vaccinations as an adjuvant therapy for this patient population.

Targeted pathways are also important and have shown added benefit for patients who express particular gene mutations. The EGFR gene mutations are one. Negative results from the NCIC CTG BR.19 trial which evaluated gefitinib in completely resected stage IB-IIIA NSCLC (only $21 \%$ of whom had EGFR mutations) have raised questions. The BR.19 trial randomized 503 patients to gefitinib versus placebo but the protocol was amended in January 2003 to allow adjuvant chemotherapy. Additionally, the trial was halted early due to the interim analysis of S0023 SWOG trial which demonstrated maintenance gefitinib was associated with worse survival than placebo after concurrent chemoradiation for stage III NSCLC. The numbers therefore in the BR.19 trial were small making any interpretations speculative at best (Thatche et al., 2005). The Randomized double-blinded trial in Adjuvant NSCLC with Tarceva (RADIANT) trial is evaluating the role of EGFR-TKI erlotinib in the adjuvant treatment of 945 patients with resected Stage IB-IIIA NSCLC whose tumors have overexpression of EGFR detected by immunohistochemistry or FISH. Patients may have up to four cycles of chemotherapy after surgery and are randomized 2:1 to erlotinib or placebo for 2 years. Patients randomized to erlotinib began within 6 months of surgery if adjuvant chemotherapy was administered or within 3 months of surgery in adjuvant chemotherapy was not given. This trial has completed accrual and full EGFR and other molecular analyses are being completed. Activation of angiogenesis is also an area of study. Despite the absence of impact of bevacizumab, a monoclonal antibody that targets angiogenesis, in adjuvant colorectal cancer, it has been demonstrated to significantly improve outcomes for patients with advanced NSCLC when combined with doublet chemotherapy as first-line therapy in both the ECOG 4599 and Avastin in Lung cancer (AVAiL) trial. Based on these positive results, the ECOG 1505 trial randomizes patients to four cycles of cisplatin-based doublet chemotherapy alone or with bevacizumab dosed at $15 \mathrm{mg} / \mathrm{kg}$ every 3 weeks.

\section{CONCLUSION}

Although a research question over a decade ago, adjuvant cisplatinbased chemotherapy is now the standard of care for patients with resected stage II and IIIA, and selected stage IB NSCLC. This is based on the results of phase III trials such as the IALT, JBR.10, and ANITA studies. Although the optimum therapy is still under investigation, and the strongest data to date show a 
trend favoring cisplatin-vinorelbine, there are many other cisplatin doublets from which oncologists are already choosing. Data from the metastatic setting extrapolated to the adjuvant setting suggest that choosing cisplatin-pemetrexed, cisplatin-docetaxel, or cisplatin-gemcitabine as an alternative to cisplatin-vinorelbine

\section{REFERENCES}

Ardizonni, A., Boni, L., Tiseo, M., Fossella, F. V., Schiller, J. H., Paesmans, M., Radosavljevic, D., Paccagnella, A., Zatloukal, P., Mazzanti, P., Bisset, D., Rosell, R., and CISCA (CISplatin versus CArboplatin) Meta-analysis Group. (2007). Cisplatin versus carboplatin-based chemotherapy in first-line treatment of advanced non-small cell lung cancer: an individual patient data meta-analysis. $J$. Natl. Cancer Inst. 99, 847-857.

Azzoli, C. G., Krug, L. M., Miller, V. A., Rizvi, N. A., Kris, M. G., Dunne, M., Farmer, A., Pizzo, B., Tyson, L., Seeger, T., Coleman, B., Moore, E., Lastinger, L., Venkatraman, E., and Rudin, C. M. (2007). A phase II tolerability study of cisplatin plus docetaxel as adjuvant chemotherapy for resected non-small cell lung cancer. J. Thorac. Oncol. 7, 638-644.

Baggstrom, M. Q., Stinchcombe, T. E., Fried, D. B., Poole, C., Hensing, T. A., and Socinski, M. A. (2007). Thirdgeneration chemotherapy agents in the treatment of advanced nonsmall cell lung cancer. J. Thorac. Oncol. 2, 845-853.

Cuffe, S., Booth, C. M., Peng, Y., Darling, G. E., Li, G., Kong, W., Mackillop, W. J., and Shepherd, F. A. (2011). Adoption of adjuvant chemotherapy for non-small cell lung cancer in the elderly: a population-based outcomes study. J. Clin. Oncol. 29, Abstr. 7012.

Douillard, J. Y., Rosell, R., De Lena, M., Carpagnano, F., Ramlau, R., Gonzáles-Larriba, J. L., Grodzki, T., Pereira, J. R., Le Groumellec, A., Lorusso, V., Clary, C., Torres, A. J., Dahabreh, J., Souquet, P. J., Astudillo, J., Fournel, P., Artal-Cortes, A., Jassem, J., Koubkova, L., His, P., Riggi, M., and Hurteloup, P. (2006). Adjuvant vinorelbine plus cisplatin versus observation in patients with completely resected stage IB-IIIA non-small-cell lung cancer (Adjuvant Navelbine International Trialist Association [ANITA]): a randomised controlled trial. Lancet Oncol. 7, 719-727.

Ettinger, D. S., Akerley, W., Bepler, G., Blum, M. G., Chang, A., Cheney, R. T., Chirieac, L. R., D'Amico, T. A., Demmy, T. L., Ganti, A. K., Govindan, R., Grannis, F. W. Jr., Jahan, T., Jahanzeb, M., Johnson, D. H., Kessinger, A., Komaki, R., Kong, F.
M., Kris, M. G., Krug, L. M., Le, Q. T., Lennes, I. T., Martins, R., O’Malley, J., Osarogiagbon, R. U., Otterson, G. A., Patel, J. D., Pisters, K. M., Reckamp, K., Riely, G. J., Rohren, E., Simon, G. R., Swanson, S. J., Wood, D. E., Yang, S. C., and NCCN NonSmall Cell Lung Cancer Panel Members. (2010). Non Small Cell Lung Cancer. J. Natl. Compr. Canc. Netw. 8, 740-801.

Ferry, D., Billingham, L., Jarrett, H., Dunlop, D., Thompson, J., Kumar, M., Skailes, G., Nicolson, M., Shah, R., Leonard, P., Chetiyawardana, A., Wells, P., Lewanski, C., Woll, P., Crosse, B., Hill, M., Pirrie, S., and O'Byrne, K. J. (2011). BROG2 Randomized phase III clinical trial of gemcitabine combined with cisplatin $50 \mathrm{mg} / \mathrm{m} 2$ (GC50) versus cisplatin $80 \mathrm{mg} / \mathrm{m} 2$ (GC80) versus carboplatin AUC 6 (BGCB6) in advanced NSCLC. J. Thorac. Oncol.

Fossella, F., Pereira, J. R., von Pawel, J., Pluzanska, A., Gorbounova, V., Kaukel, E., Mattson, K. V., Ramlau, R., Szczesna, A., Fidias, P., Millward, M., and Belani, C. P. (2003). Randomized, multinational, phase III study of docetaxel plus platinum combinations versus vinorelbine plus cisplatin for advanced non small cell lung cancer: the TAX 326 study group. J. Clin. Oncol. 21, 3016-3024.

Fruh, M., Rolland, E., Pignon, J. P., Seymour, L., Ding, K., Tribodet, H., Winton, T., Le Chevalier, T., Scagliotti, G. V., Douillard, J. Y., Spiro, S., and Shepherd, F. A. (2008). Pooled analysis of the effect of age on adjuvant cisplatin-based chemotherapy for completely resected non-small cell lung cancer. J. Clin. Oncol. 26, 3573-3581.

Gu, F., Strauss, G. M., and Wisnivesky, J. P. (2011). "Platinumbased adjuvant chemotherapy in elderly patients with non-small cell lung cancer in the SEER-Medicare database: comparison between carboplatin and cisplatin-based regimens," in Abstract presented at the American Society of Clinical Oncology, Chicago, IL.

IALT. (2004). The International Adjuvant Lung Cancer Trial Collaborative Group: cisplatin based adjuvant chemotherapy in patients with 6(Suppl. 2), S274.

is reasonable. Studies are underway to examine these regimens further in this setting, with a strong focus on utilization of biomarkers to select the best drugs for each patient. These future studies will hopefully lead the way toward better survival for patients with resectable NSCLC.

completely resected nonsmall cell lung cancer. N. Engl. J. Med. 350, 351-360.

Kohler, B. A., Ward, E., McCarthy, B. J., Schymura, M. J., Ries, L. A., Eheman, C., Jemal, A., Anderson, R. N., Ajani, U. A., and Edwards, B. K. (2011). Annual Report to the Nation on the Status of Cancer, 1975-2007, featuring tumors of the brain and other nervous system. J. Natl. Cancer Inst. 103, 1-23.

Kreuter, M., Vansteenkiste, J., Griesinger, F., Hoffmann, H., Dienemann, H., De Leyn, P., and Thomas, M. (2011). "Trial on the refinement of early non-small cell lung cancer. Adjuvant chemotherapy with pemetrexed and cisplatin versus vinorelbine and cisplatin: the TREAT protocol," Abstract presented at the American Society of Clinical Oncology, Chicago, IL.

Non-Small Cell Lung Cancer Collaborative Group. (1995). Chemotherapy in non-small cell lung cancer: a meta-analysis using updated data on individual patients from 52 randomised clinical trials. BMJ 311 , 899-909.

Olaussen, K. A., Dunant, A., Fouret, P., Brambilla, E., André, F., Haddad, V., Taranchon, E., Filipits, M., Pirker, R., Popper, H. H., Stahel, R., Sabatier, L., Pignon, J. P., Tursz, T., Le Chevalier, T., Soria, J. C., and IALT Bio Investigators. (2006). DNA repair by ERCC1 in non-small-cell lung cancer and cisplatin-based adjuvant chemotherapy. N. Engl. J. Med. 355, 983-991.

Pignon, J. P., Tribodet, H., Scagliotti, G. V., Douillard, J. Y., Shepherd, F. A., Stephens, R. J., Dunant, A., Torri, V., Rosell, R., Seymour, L., Spiro, S. G., Rolland, E., Fossati, R., Aubert, D., Ding, K., Waller, D., Le Chevalier, T., and LACE Collaborative Group. (2008). Lung adjuvant cisplatin evaluation: a pooled analysis by the LACE Collaborative Group. J. Clin. Oncol. 26, 3552-3559.

Scagliotti, G. V., Fossati, R., Torri, V., Crinò, L., Giaccone, G., Silvano, G., Martelli, M., Clerici, M., Cognetti, F., Tonato, M., and For the Adjuvant Lung Project Italy/European Organisation for Research Treatment of CancerLung Cancer Cooperative Group Investigators. (2003). Randomized study of adjuvant chemotherapy for completely resected stage 1 , 11, or IIIA non-small cell lung cancer. J. Natl. Cancer Inst. 95, 1453-1461.

Scagliotti, G. V., Parikh, P., von Pawel, J., Biesma, B., Vansteenkiste, J., Manegold, C., Serwatowski, P., Gatzemeier, U., Digumarti, R., Zukin, M. Lee, J. S., Mellemgaard, A., Park, K., Patil, S., Rolski, J., Goksel, T., de Marinis, F., Simms, L., Sugarman, K. P., and Gandara, D. (2008). Phase III study comparing cisplatin plus gemcitabine with cisplatin plus pemetrexed in chemotherapy-naïve patients with advanced stage non small cell lung cancer. J. Clin. Oncol. 26, 3543-3551.

Schiller, J. H., Harrington, D., Belani, C. P., Langer, C., Sandler, A., Krook, J., Zhu, J., Johnson, D. H., and Eastern Cooperative Oncology Group. (2002). Comparison of four chemotherapy regimens for advanced non-small cell lung cancer. N. Engl. J. Med. 346, 92-98.

Siegel, R., Ward, E., Brawley, O., and Jemal, A. (2011). Cancer Statistics: the impact on eliminating socioeconomic and racial disparities on premature cancer deaths. CA Cancer J. Clin. 61, 212-236.

Stewart, L. A., Burdett, S., Tierney, J. F., Pignon, J., and On Behalf of the NSCLC Collaborative Group. (2007). Surgery and adjuvant chemotherapy (CT) compared to surgery alone in non-small cell lung cancer (NSCLC): a metaanalysis using individual patient data (IPD) from randomized clinical trials (RCT). J. Clin. Oncol. 25, 397s.

Strauss, G. M., Herndon, J. E. II, Maddaus, M. A., Johnstone, D. W., Johnson, E. A., Harpole, D. H., Gillenwater, H. H., Watson, D. M., Sugarbaker, D. J., Schilsky, R. L., Vokes, E. E., Green, M. R. (2008). Adjuvant paclitaxel plus carboplatin compared with observation in stage 1B non small cell lung cancer: CALGB 9633 with the Cancer and Leukemia Group B Radiation Therapy Oncology Group and the North Central Cancer Treatment Group Study Groups. J. Clin. Oncol. 26, 5043-5051.

Thatche, R. N., Chang, A., Parikh, P., Rodrigues Pereira, J., Ciuleanu, T., 
von Pawel, J., Thongprasert, S., Tan, E. H., Pemberton, K., Archer, V., and Carroll, K. (2005). Gefitinib plus best supportive care in previously treated patients with refractory advanced non-small cell lung cancer: results from a randomized, placebo controlled multicenter study (Iressa Survival Evaluation in Lung Cancer). Lancet 366, 1527-1537.

Wakelee, H., Dahlberg, S. E., Keller, S. M., Gandara, D. R., Graziano, S. L., Leighl, N. B., Adjei, A. A., Schiller, J. H., and Eastern Cooperative Oncology Group. (2010). "Interim report of on-study demographics and toxicity from E1505, a phase III randomized trial of adjuvant chemotherapy with or without bevacizumab for completely resected early-stage non-small cell lung cancer," in Abstract presented at the American Society of Clinical Oncology, Chicago, IL.

Waller, D., Peake, M. D., Stephens, R. J., Gower, N. H., Milroy, R., Parmar, M. K., Rudd, R. M., and Spiro, S. G. (2004). Chemotherapy for patients with non-small cell lung cancer: the surgical setting of the Big Lung Trial. Eur. J. Cardiothorac. Surg. 26, 173-182.

Winton, T., Livingston, R., Johnson, D., Rigas, J., Johnston, M., Butts, C., Cormier, Y., Goss, G., Inculet, R., Vallieres, E., Fry, W., Bethune, D., Ayoub, J., Ding, K., Seymour, L., Graham, B., Tsao, M. S., Gandara, D., Kesler, K.,
Demmy, T., Shepherd, F., National Cancer Institute of Canada Clinical Trials Group, and National Cancer Institute of the United States Intergroup JBR.10 Trial Investigators. (2005). Vinorelbine plus cisplatin vs. observation in resected non-smallcell lung cancer. N. Engl. J. Med. 352, 2589-2597.

Conflict of Interest Statement: The authors declare that the research was conducted in the absence of any commercial or financial relationships that could be construed as a potential conflict of interest.

Received: 11 September 2011; paper pending published: 11 October 2011; accepted: 03 November 2011; published online: 24 November 2011.

Citation: Patel MI and Wakelee HA (2011) Adjuvant chemotherapy for early stage non-small cell lung cancer. Front. Oncol. 1:45. doi: 10.3389/fonc.2011.00045

This article was submitted to Frontiers in Thoracic Oncology, a specialty of Frontiers in Oncology.

Copyright @2011 Patel and Wakelee. This is an openaccess article subject to a non-exclusive license between the authors and Frontiers Media SA, which permits use, distribution and reproduction in other forums, provided the original authors and source are credited and other Frontiers conditions are complied with. 